\title{
On the Reflected Geometric Brownian Motion with Two Barriers
}

\author{
Lidong Zhang ${ }^{1}$, Ziping Du ${ }^{2}$ \\ ${ }^{1}$ Department of Mathematics, Tianjin University of Science\&Technology, Tianjin, China \\ ${ }^{2}$ College of Economics\&Management, Tianjin University of Science\&Technology, Tianjin, China \\ E-mail: zhanglidong1979@yahoo.com.cn,dusx@tust.edu.cn \\ Received January 28, 2009; revised March 3, 2010; accepted April 7, 2010
}

\begin{abstract}
In this paper, we are concerned with Reflected Geometric Brownian Motion (RGBM) with two barriers. And the stationary distribution of RGBM is derived by Markovian infinitesimal Generator method. Consequently the first passage time of RGBM is also discussed.
\end{abstract}

Keywords: Geometric Brownian Motion, Stationary Distribution, First Passage Time

\section{Introduction}

We consider a finite-capacity fluid queue, the level of which at time $t$ is denoted by $Z_{\mathrm{t}}$. And $Z_{\mathrm{t}}$ satisfies the following differential equation:

$$
\left\{\begin{array}{l}
d Z_{t}=\left(\alpha_{1}-\alpha_{2}\right) Z_{t} d t+\sigma Z_{t} d B_{t}+d L_{t}-d U_{t} \\
Z_{0}=x \in[a, d]
\end{array}\right.
$$

This model shows fluid arrives into this queue at rate $\alpha_{1} Z_{t}$ and leaves the queue at rate $\alpha_{2} Z_{t}$. This fluid level can be also varied by a local variance function $\sigma Z_{t}$ and a standard Brownian motion $B_{\mathrm{t}} . L_{t}$ and $U_{t}$ are nondecreasing processes, interfering only when $Z_{\mathrm{t}}$ hits $a$ or $d$ and make $Z_{\mathrm{t}}$ vary between $a$ and $d$.

In particular, when $a \rightarrow 0^{+}$and $d \rightarrow+\infty, L_{t}$ and $U_{t}$ disappear. Then the process $Z_{t}$ becomes Geometric Brownian Motion. So we call $Z_{t}$ determined by (1) Reflected Geometric Brownian Motion(RGBM).

Speak precisely, we are concerned with RGBM $Z=\left\{Z_{t}, t \geq 0\right\}$ with two barriers $a$ and $d(d>a>0)$, which is defined by

$$
\left\{\begin{array}{l}
d Z_{t}=\mu Z_{t} d t+\sigma Z_{t} d B_{t}+d L_{t}-d U_{t} \\
Z_{0}=x \in[a, d]
\end{array}\right.
$$

where $B=\left\{B_{\mathrm{t}}, t \geq 0\right\}$ is a standard Brownian Motion, $\sigma>0, \mu$ and $\sigma$ are constants and satisfy $\mu \neq \frac{\sigma^{2}}{2}$.
Moreover, the processes $L$ and $U$ are uniquely determined by the following property $[1,2]$ :

1) Both $L$ and $U$ are continuous nondecreasing processes with $L_{0}=U_{0}=0$;

2) $L$ and $U$ increase only when $Z_{t}=a$ and $Z_{t}=d$; respectively, i.e.,

$$
\int_{0}^{t} I_{\left\{Z_{s}=a\right\}} d L_{s}=L_{t}, \int_{0}^{t} I_{\left\{Z_{s}=d\right\}} d U_{s}=U_{t}, \text { for } t \geq 0 .
$$

According to the theory of stochastic differential equation, (2) is equivalent to

$$
Z_{t}=\mathrm{x}+\mu \int_{0}^{\mathrm{t}} Z_{\mathrm{s}} d s+\sigma \int_{0}^{\mathrm{t}} Z_{\mathrm{s}} d B_{s}+L_{t}-U_{t}
$$

Such a process is a regenerative Markov process with state space $[a, d]$ compact. Then it has a unique stationary distribution $[1,3,4]$. In the coming section, our objective is to derive the stationary distribution and give an expression for the Laplace Transform of the first passage time of RGBM $Z=\left\{Z_{t}, t \geq 0\right\}$ by the method in [5-7].

\section{Main Results on RGBM}

\subsection{On the Stationary Distribution of RGBM}

In this section, we firstly give a Lemma on the stationary distribution of the reflected process $Z=\left\{Z_{\mathrm{t}}\right\}$ with two-sided barriers and omit its proof.

Lemma 2.1 Let $Z$ be the RGBM defined by (2) (or(3)). Then, as a Markov process, the stationary distribution $\pi(\cdot)$ of the process must satisfy the following equation 


$$
\begin{aligned}
& \int_{a}^{d} A f(x) \pi(d x)=\int_{a}^{d}\left\{\frac{\sigma^{2} x^{2}}{2} f^{\prime \prime}(x)+\mu x f^{\prime}(x)+f^{\prime}(a) \gamma(x)\right. \\
& \left.-f^{\prime}(d) \beta(x)\right\} \pi(d x)=0
\end{aligned}
$$

where $\quad \gamma(x)=\lim _{t \rightarrow \infty} \frac{d E_{x} L_{t}}{d t}, \beta(x)=\lim _{t \rightarrow \infty} \frac{d E_{x} U_{t}}{d t} \quad$ and $f \in C_{b}^{2}([a, d])$ which denotes the space of all bounded continuous functions having twicely continuous derivatives on $[a, d]$.

Proof. See similar argument in [1].

Suppose $\pi(d x)=p(x) d x$ be a probability distribution on $[a, d]$ and satisfies that

$$
\int_{a}^{d} A f(x) p(x) d x=0
$$

for $\forall f \in C_{b}^{2}([a, d])$.

Define $A_{1} f(x)=\frac{\sigma^{2} x^{2}}{2} f^{\prime \prime}(x)+\mu x f^{\prime}(x)$, then by (4) and (5) it is equivalent to the following equation (Note that $\left.\int_{\mathrm{a}}^{\mathrm{d}} p(x) d x=1\right)$

$$
\int_{a}^{d} A_{1} f(x) p(x) d x+f^{\prime}(a) \gamma-f^{\prime}(d) \beta=0
$$

where $\gamma=\int_{\mathrm{a}}^{\mathrm{d}} \gamma(x) p(x) d x$ and $\beta=\int_{\mathrm{a}}^{\mathrm{d}} \beta(x) p(x) d x$.

On one hand, $\gamma$ and $\beta$ can be computed by the same method in [5].

Proposition 2.1 Choose $f_{1}=x^{-\frac{2 \mu}{\sigma^{2}}}$ and $f_{1}=x^{-1}$, then they respectively satisfy the following equations,

$$
\begin{aligned}
& A_{1} f_{1}(x)=0, x \in[a, d] \\
& A_{1} f_{2}(x)=1, x \in[a, d]
\end{aligned}
$$

Then we have

$$
\beta=\frac{\mu-\frac{\sigma^{2}}{2}}{\mathrm{~d}^{\frac{2 \mu}{\sigma^{2}}-1}-\mathrm{a}^{\frac{2 \mu}{\sigma^{2}}-1}} \mathrm{~d}^{\frac{2 \mu}{\sigma^{2}}}, \gamma=\frac{\mu-\frac{\sigma^{2}}{2}}{\mathrm{~d}^{\frac{2 \mu}{\sigma^{2}}-1}-\mathrm{a}^{\frac{2 \mu}{\sigma^{2}}-1}} \mathrm{a}^{\frac{2 \mu}{\sigma^{2}}} .
$$

Proof. A straightforward calculation.

On the other hand, since $p(x)$ satisfies that for all $f \in C_{b}^{2}([a, d])$,

$$
\int_{a}^{d}\left[\frac{\sigma^{2} x^{2}}{2} f^{\prime \prime}(x)+\mu x f^{\prime}(x)\right] p(x) d x+f^{\prime}(a) \gamma-f^{\prime}(d) \beta=0
$$

By twice integral changes, the above equation becomes that

$$
\begin{aligned}
& \int_{a}^{d}\left[\frac{\sigma^{2} x^{2}}{2} p^{\prime \prime}(x)+\left(2 \sigma^{2}-\mu\right) x p^{\prime}(x)+\left(\sigma^{2}-\mu\right) p(x)\right] \\
& f(x) d x+\left[\frac{\sigma^{2} x^{2}}{2} f^{\prime}(x) p(x)+\mu x p(x) f(x)\right]_{a}^{d}
\end{aligned}
$$

$$
\begin{aligned}
& -\left[\left(\sigma^{2} x p(x)+\frac{\sigma^{2} x^{2}}{2} p^{\prime}(x)\right) f(x)\right]_{a}^{d} \\
& +f^{\prime}(a) \gamma-f^{\prime}(d) \beta=0
\end{aligned}
$$

i.e.

$$
\begin{aligned}
& \int_{a}^{d}\left[\frac{\sigma^{2} x^{2}}{2} p^{\prime \prime}(x)+\left(2 \sigma^{2}-\mu\right) x p^{\prime}(x)+\left(\sigma^{2}-\mu\right) p(x)\right] \\
& f(x) d x+f(d)\left[\mu d p(d)-\sigma^{2} d p(d)+\frac{\sigma^{2} d^{2}}{2} p^{\prime}(d)\right] \\
& -f(a)\left[\mu a p(a)-\sigma^{2} a p(a)+\frac{\sigma^{2} a^{2}}{2} p^{\prime}(a)\right] \\
& +f^{\prime}(d)\left[\frac{\sigma^{2} d^{2}}{2} p(d)-\beta\right] \\
& -f^{\prime}(a)\left[\frac{\sigma^{2} a^{2}}{2} p(a)-\gamma\right]=0
\end{aligned}
$$

Assume that $f \in C_{b}^{2}([a, d])$, satisfying that $f(d)=0$, $f^{\prime}(d)=0$, and $P(x)$ satisfies $\frac{\sigma^{2} a^{2}}{2} p(a)=\gamma$ and $\mu a p(a)-\sigma^{2} a p(a)+\frac{\sigma^{2} a^{2}}{2} p^{\prime}(a)=0$, then it follows from (7) that

$$
\begin{aligned}
& \int_{a}^{d}\left[\frac{\sigma^{2} x^{2}}{2} p^{\prime \prime}(x)+\left(2 \sigma^{2}-\mu\right) x p^{\prime}(x)+\left(\sigma^{2}-\mu\right) p(x)\right] \\
& f(x) d x=0
\end{aligned}
$$

Summarizing the discussion, we get the following theorem.

Theorem 2.1 $p(x)=\frac{\frac{2 \mu}{\sigma^{2}}-1}{d^{\frac{2 \mu}{\sigma^{2}}-1}-a^{\frac{2 \mu}{\sigma^{2}}-1}} x^{\frac{2 \mu}{\sigma^{2}-2}}$ is the solution of

$$
\left\{\begin{array}{l}
\frac{\sigma^{2} x^{2}}{2} p^{\prime \prime}(x)+\left(2 \sigma^{2}-\mu\right) x p^{\prime}(x)+\left(\sigma^{2}-\mu\right) p(x)=0 \\
\frac{\sigma^{2} a^{2}}{2} p(a)=\gamma \\
\int_{a}^{d} p(x) d x=1
\end{array}\right.
$$

Then for all $f \in C_{b}^{2}([a, d])$ satisfying $f(d)=0$, $f^{\prime}(d)=0$, (5) holds, i.e. $\int_{a}^{d} A f(x) p(x) d x=0$.

Furthermore (5) holds for all $f \in C_{b}^{2}([a, d])$. This implies that $\pi(d x)=p(x) d x$ is a stationary distribution of the corresponding Markov process $Z=\left\{Z_{t}, t \geq 0\right\}$.

Remark 2.1 This theorem is a standard application of renewal theorems, so we sketch its proof. 
Thus $p(x)=\frac{\frac{2 \mu}{\sigma^{2}}-1}{d^{\frac{2 \mu}{\sigma^{2}}-1}-a^{\frac{2 \mu}{\sigma^{2}}-1}} x^{\frac{2 \mu}{\sigma^{2}}-2}, x \in[a, d]$ is the density of the stationary distribution of RGBM. Finally we will give an expression for the Laplace transform of the first passage time of RGBM.

\subsection{On the First Passage Time of RGBM}

In this section, we consider Equation (2). Let $y \in[a, d]$, define the first passage time by $T(y):=\inf \left\{t \geq 0: Z_{t}=y\right\}$, with the usual convention $\inf \varnothing=\infty$. On the other hand, suppose $\lambda>0$, for $f \in C_{b}^{2}([a, d])$, define a operator

$$
A^{(\lambda)} f(x)=\frac{\sigma^{2} x^{2}}{2} f^{\prime \prime}(x)+\mu x f^{\prime}(x)-\lambda f(x), x \in[a, d]
$$

Finally we are going to give the expression of the Laplace transform of $T(y)$.

Theorem 2.2. For $x \in[a, d]$ and $\lambda>0$, then

$$
\begin{aligned}
& E_{x}\left(e^{-\lambda T(y)}\right)=\frac{f_{1}^{\lambda}(x)}{f_{1}^{\lambda}(y)}, x \leq y \leq d \\
& E_{x}\left(e^{-\lambda T(y)}\right)=\frac{f_{2}^{\lambda}(x)}{f_{2}^{\lambda}(y)}, a<y \leq x
\end{aligned}
$$

where

$$
\begin{aligned}
& f_{1}^{\lambda}(x)=x^{\frac{\sigma^{2}-2 \mu+\sqrt{\left(\sigma^{2}-2 \mu\right)^{2}+8 \lambda \sigma^{2}}}{2 \sigma^{2}}}+c_{1} x \frac{\sigma^{2}-2 \mu-\sqrt{\left(\sigma^{2}-2 \mu\right)^{2}+8 \lambda \sigma^{2}}}{2 \sigma^{2}}, \\
& f_{2}^{\lambda}(x)=x \frac{\sigma^{2}-2 \mu+\sqrt{\left(\sigma^{2}-2 \mu\right)^{2}+8 \lambda \sigma^{2}}}{2 \sigma^{2}}+c_{2} x \frac{\sigma^{2}-2 \mu-\sqrt{\left(\sigma^{2}-2 \mu\right)^{2}+8 \lambda \sigma^{2}}}{2 \sigma^{2}},
\end{aligned}
$$

and

$$
\begin{aligned}
& C_{1}=\frac{\left(\sqrt{\left(\sigma^{2}-2 \mu\right)^{2}+8 \lambda \sigma^{2}}+\left(\sigma^{2}-2 \mu\right)\right)^{2}}{8 \lambda \sigma^{2}} a^{2 \sqrt{\left(\sigma^{2}-2 \mu\right)^{2}+8 \lambda \sigma^{2}}}, \\
& C_{2}=\frac{\left(\sqrt{\left(\sigma^{2}-2 \mu\right)^{2}+8 \lambda \sigma^{2}}+\left(\sigma^{2}-2 \mu\right)\right)^{2}}{8 \lambda \sigma^{2}} d^{2 \sqrt{\left(\sigma^{2}-2 \mu\right)^{2}+8 \lambda \sigma^{2}}} .
\end{aligned}
$$

Proof. Let $h(t, x)=e^{-\lambda t} f(x)$ for $f \in C_{b}^{2}([a, d])$. Then applying It $\hat{o}$ formula for $h(t, x)$, we have

$$
\begin{aligned}
& h\left(t, Z_{t}\right)=h\left(0, Z_{0}\right)+\int_{0}^{t} \frac{\partial h}{\partial s}\left(s, Z_{s}\right) d s+\int_{0}^{t} \frac{\partial h}{\partial Z_{s}}\left(s, Z_{s}\right) d Z_{s} \\
& +\frac{1}{2} \int_{0}^{t} \frac{\partial^{2} h}{\partial Z_{s}^{2}}\left(s, Z_{s}\right) d<Z, Z>_{s} \\
& =\int_{0}^{t} e^{-\lambda s}\left[\frac{\sigma^{2} Z_{s}^{2}}{2} f^{\prime \prime}\left(Z_{s}\right)+\mu Z_{s} f^{\prime}\left(Z_{s}\right)-\lambda f\left(Z_{s}\right)\right] d s
\end{aligned}
$$

$$
\begin{aligned}
& +f\left(Z_{0}\right)+\int_{0}^{t} e^{-\lambda s} f^{\prime}\left(Z_{s}\right) d L_{s}-\int_{0}^{t} e^{-\lambda s} f^{\prime}\left(Z_{s}\right) d U_{s} \\
& +\sigma \int_{0}^{t} e^{-\lambda s} Z_{s} f^{\prime}\left(Z_{s}\right) d B_{s} \\
& =\int_{0}^{t} e^{-\lambda s} A^{(\lambda)} f\left(Z_{s}\right) d s+f\left(Z_{0}\right)+f^{\prime}(a) \int_{0}^{t} e^{-\lambda s} d L_{s} \\
& -f^{\prime}(d) \int_{0}^{t} e^{-\lambda s} d U_{s}+\sigma \int_{0}^{t} e^{-\lambda s} Z_{s} f^{\prime}\left(Z_{s}\right) d B_{s}
\end{aligned}
$$

The last equation holds, for $L_{t}$ and $U_{t}$ increase only when $Z_{\mathrm{t}}=a$ and $Z_{\mathrm{t}}=d$. Let $T<\infty$ be a stopping time and $x \in[a, d]$. It follows from martingale optional theorem, that

$$
\begin{aligned}
& E_{x}\left[e^{-\lambda T} f\left(Z_{T}\right)\right] \\
& =f(x)+f^{\prime}(a) E_{x}\left[\int_{0}^{T} e^{-\lambda s} d L_{s}\right] \\
& -f^{\prime}(d) E_{x}\left[\int_{0}^{T} e^{-\lambda s} d U_{s}\right]+E_{x}\left[\int_{0}^{T} e^{-\lambda s} A^{(\lambda)} f\left(Z_{s}\right) d s\right]
\end{aligned}
$$

In particular, take $T=T(y)$ for $\mathrm{y} \in[\mathrm{a}, \mathrm{d}]$, and note that

$$
\int_{0}^{T(y)} e^{-\lambda s} d U_{s}=0, \text { for } \quad x \leq y \leq d,
$$

and

$$
\int_{0}^{T(y)} e^{-\lambda s} d L_{s}=0, \text { for } \quad a \leq y \leq x,
$$

Then

$$
\begin{aligned}
& E_{x}\left(e^{-\lambda T(y)} f\left(Z_{T(y)}\right)\right) \\
& =f(x)+E_{x}\left(\int_{0}^{T(y)} e^{-\lambda s} A^{(\lambda)} f\left(Z_{s}\right) d s\right) \\
& +f^{\prime}(a) E_{x}\left(\int_{0}^{T(y)} e^{-\lambda s} d L_{s}\right), \text { for } \quad x \leq y \leq d,
\end{aligned}
$$

and

$$
\begin{aligned}
& E_{x}\left(e^{-\lambda T(y)} f\left(Z_{T(y)}\right)\right) \\
& =f(x)+E_{x}\left(\int_{0}^{T(y)} e^{-\lambda s} A^{(\lambda)} f\left(Z_{s}\right) d s\right) \\
& -f^{\prime}(d) E_{x}\left(\int_{0}^{T(y)} e^{-\lambda s} d U_{s}\right), \text { for } \quad a \leq y \leq x,
\end{aligned}
$$

Replace $f$ by $f_{1}^{\lambda}$ in (14) and by $f_{2}^{\lambda}$ in (15), we immediately get (10) and (11) by $Z_{T(y)}=y$, $f_{1}^{\lambda}{ }^{\prime}(a)=0$ and $f_{2}^{\lambda}{ }^{\prime}(d)=0$. Thus the Proof of the theorem is completed.

\section{Conclusions}

This paper studies Reflected Geometric Brownian Motion (RGBM) with two barriers. Both the stationary distribution and Laplace transform of the first passage time of RGBM are derived. The studies for RGBM have not only practical significance, but also give an important 
result in theory of stochastic process.

\section{Acknowledgements}

This research is supported by the National Natural Science foundation of China (Grant No.70671074) and the Research Foundation of Tianjin university of Science and technology (Grant No.20080207). The authors would like to thank an anonymous referee for his constructive comments and suggestions on the first version of the manuscript.

\section{References}

[1] J. M. Harrison, "Brownian motion and stochastic flow systems,” John Wiley \& Sons Ltd., New York, 1986.

[2] X. Y. Xing, W. Zhang and Y. J. Wang, "The Stationary Distributions of Two Classes of Reflected Ornstein-Uhlenbeck Processes," Journal of Applied Prob- ability, Vol. 46, No. 3, 2009, pp. 709-720.

[3] S. Asmussen and O. Kella, "A Multi-Dimensional Martingale for Markov Additive Process and its Applications," Advances in Applied Probability, Vol. 32, No. 2, 2000, pp. 376-393.

[4] S. Either and T. Kurtz, "Markov Processes: Characterization and Convergences,” John Wiley Sons, New York, 1986.

[5] L. J. Bo, L. D. Zhang and Y. J. Wang, "On the First Passage Times of Reflected O-U Processes with Two-Sided Barriers,” Queueing Systems, Vol. 54, No. 4, December 2006, pp. 313-316.

[6] V. Linetsky, "On the Transition Densities for Reflected Diffusions,” Advances in Applied Probability, Vol. 37, No. 2, 2005, pp. 435-460.

[7] L. Rabehasaina and B. Sericola, “A Second-Order Markov-Modulated Fluid Queue with Linear Service Rate,” Journal of Applied Probability, Vol. 41, No. 3, 2004, pp. 758-777. 